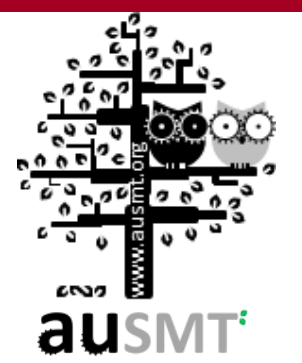

\title{
EDITORIAL
}

\section{Smart Technology for the Older Adults to Facilitate Independent Living and Social Participation}

\section{Yeh-Liang Hsu}

Department of Mechanical Engineering, Yuan Ze University, Taoyuan 32003, Taiwan

(Published on line 1 December 2015)

mehsu@saturn.yzu.edu.tw

DOI: 10.5875 /ausmt.v5i4.1064

"W e are in the midst of two striking trends: Wide-spread population aging and rapid diffusion of technology. Both phenomena are very new in human history" [1]. It is only natural to consider developing technology to help solving the various problems in the aging society. Gerontechnology is an immerging field aiming at "designing technology and environment for independent living and social participation of older persons in good health, comfort and safety" [2]. Possible application domains include health, housing, mobility, communication, leisure, and work [3]. Gerontechnology is interdisciplinary in nature and represents an important opportunity for industry, facing the aged society. On the other hand, gerontechnology research is only valuable if it can be realized into products or services that benefit older persons and their caregivers.

This special issue publishes seven papers on development of smart technology for older adults to facilitate independent living and social participation. The papers are highlighted briefly as follows.

The paper entitled "Design and evaluation of the walking cane handle grip" co-authored by Kimitomo Taniguchi and Atsuo Takanishi seeks to optimize the grip shape of canes used as walking aids to reduce the dorsiflexion of the wrist joint. The stability of the prototype grip is evaluated using a floor reaction force gauge based on a Nintendo Wii Balance Board, and the prototype showed good weight shift, and improved stability of weight transfer during walking.

The paper entitled "Finite element estimation of pressure distribution inside the trunk on a mattress" coauthored by Shigekazu Ishihara, Keiko Ishihar, and Mitsuo Nagamachi attempts to model the body and estimate the pressure on its transverse plane. The computation was based on a non-linear finite element method with hyperelastic materials, such as muscle, skin and fat. Because the model simulates different tissues, pressure inside the trunk can also be estimated.

The paper entitled "Smart Home Strategies for User-Centered Functional Assessment of Older Adults" co-authored by Vijay Kumar Ravishankar, Winslow Burleson, and Diane Mahoney presents strategies for user-centric approaches to identify the technical and design challenges of developing, deploying, and using functional assessment systems in homes occupied by senior citizens. The results suggest strategies for usercentered functional assessment and assistive technology design and implementation, providing information capture, analysis, and delivery of in-home functional assessment that has the potential to support aging in place.

The paper entitled "Design Considerations for Technology Interventions to Support Social and Physical Wellness for Older Adults with Disability" co-authored by Jenay M. Beer, Tracy L. Mitzner, Rachel E. Stuck, and Wendy A. Rogers provides an overview of constructs related to social and physical wellness, as well as evidence-based interventions effective with older populations. Considerations for how interventions may need to be developed or modified to be efficacious for this population segment. Technology may be a key component in adopting interventions, particularly tele-technologies.

The paper entitled "Finite Element Analysis and Empirical Analysis of a Cost-effective Pressure UlcerPreventing Mattress" co-authored by Yi-Horng Lai and Lan-Yuen Guo evaluates a new passive mattress. Finite element analysis was used to determine the curved surface design of a mushroom-shaped support. Experimental results demonstrated that the new passive mattress can provide an effective and economical solution for preventing pressure ulcer. 
The paper entitled "INVITATION: An Elderly Friendly ICT-enabled Interactive Installation to Promote Social Participation" authored by Hsiang-Lan Shih, Fang Ting Huang, Tsang-Gang Lin, and Chun-Ting Lee of Industrial Technology Research Institute, Taiwan reports the design and production of an innovative recreation installation featuring elderly friendly technologies to enhance the social lives of elderly Chinese people. The contents are composed of modernized visualization elements of traditional Chinese painting designed to engage elderly Chinese people and promote interaction and participation.

The paper entitled "Analysis of Personal Life Patterns Using Accelerometer-Based Wearable Devices" co-authored by Yeh-Liang Hsu, Yi-Fan Jiang, Ching-Hung Lin, Tsai-Ya Lai, and Cheng-Li Chang analyzes personal life patterns using accelerometer-based wearable devices. Two features derived from G-sensor motion signals, average cadence (step count divided by time) and ratio of high $\mathrm{G}$ value (outside the range of $0.5 \mathrm{~g}^{\sim} 1.5 \mathrm{~g}$ ), were used to classify physical activities into four intensity levels (sedentary, light, moderate, hard). Physical activity levels (PAL) and personal life patterns of the participants were then derived.

\section{References}

[1] D. C. Burdick and S. Kwon, Gerotechnology: research and practice in technology and aging: a textbook and reference for multiple disciplines, Springer Publishing Company, 2004.

[2] N. Charness, S. J. Czaja, A. D. Fisk, and W. Rogers, "Why gerontechnology?" Gerontechnology, vol. 1, no. 2, pp. 85-87, 2001.

[3] J. L. Fozard and W. D. Kearns, "Persuasive GERONtech-nology: Reaping technology's coaching benefits at older age," In Persuasive Technology, pp. 199-202, Springer Berlin Heidel-berg, 2006.

doi: $\underline{10.1007 / 1175549430}$
Yeh-Liang Hsu is a professor of the Department of Mechanical Engineering, Yuan Ze University, Taiwan. He received his Ph.D. in mechanical engineering from Stanford University. In recent years, Professor Hsu transferred his research interest in mechanical design into gerontechnology and established the Gerontechnology Research Center in Yuan Ze University. He is now the President of Chinese Society of Gerontechnology and Service and Vice President / IT Director of the International Society. 